\title{
Gerontología: un mirada bibliométrica de las citaciones clásicas en Web Of Science (1975-2018)
}

\section{Gerontology: a bibliometric look at classical citations in Web Of Science (1975-2018)}

\author{
Ingrid Fergusson-Cárdenas \\ Claudio Rojas-Jara \\ Marcelo Piña-Morán \\ Universidad Católica del Maule, Chile \\ Roberto Polanco-Carrasco \\ Cuadernos de Neuropsicología / Panamerican Journal of Neuropsychology, Chile \\ María Gladys Olivo-Viana \\ Universidad Católica del Maule, Chile
}

Rec (22 de Diciembre de 2019) Acept (26 de Enero de 2020)

\begin{abstract}
Resumen
La gerontología es comprendida como un enfoque interdisciplinario que aborda el proceso de envejecimiento y vejez. El presente artículo caracteriza la evolución de la investigación en el campo gerontológico durante los últimos 44 años a través de un análisis bibliométrico de los trabajos de mayor impacto en el área. Se revisaron 94 publicaciones de la colección principal de la Web of Science (WoS) de Thomson Reuters en el periodo 1975-2018. Se analizan las publicaciones y su evolución longitudinal, el acoplamiento de documentos clásicos, áreas de investigación, autores y co-autorías, revistas y países. Se concluye que Estados Unidos es el país que reúne la mayor cantidad de publicaciones, citas y revistas de difusión de textos clásicos.

Palabras clave: Gerontología; Bibliometría; h-clásico; Citaciones clásicas.
\end{abstract}

\begin{abstract}
Gerontology is understood as an interdisciplinary approach that addresses the process of aging and old age. The present article characterizes the evolution of research in the gerontological field during the last 44 years through a bibliometric analysis of the works of greatest impact in the area. Ninety-four publications were reviewed from the main collection of the Web of Science (WoS) by Thomson Reuters in the period 1975-2018. The publications and their longitudinal evolution, the coupling of classic documents, research areas, authors and co-authors, journals and countries are analyzed. It is concluded that the United States is the country with the largest number of publications, citations and journals disseminating classical texts. Keywords: Gerontology; Bibliometrics; $h$-classics; Classics citations.
\end{abstract}

Correspondencia: Ingrid Fergusson-Cárdenas; Facultad de Ciencias de la Salud, Departamento de Psicología, Universidad Católica del Maule. Dirección: Av. San Miguel 3605, Talca, Chile. Teléfono: +56 71 2203398; Email: ifergusson@ucm.cl 


\section{Introducción}

El término gerontología surge del griego geron que significa anciano o senescente y logos que es un estudio o tratado (Orellana, 2013). Desde un punto epistemológico se discute si la gerontología constituye una disciplina o profesión autónoma o más bien se trata de un enfoque (Paola, 2019; Piña-Morán, Olivo-Viana, MartínezMaldonado, \& Mendoza-Núñez, 2018). Para efectos de esta investigación, se propone la gerontología como un enfoque interdisciplinario que aborda el proceso de envejecimiento y vejez en sus dimensiones biológicas, psicológicas, sociales, culturales, históricas, políticas, educativas y económicas. Además, se ha de considerar que son tres los temas centrales que definen la misión y el propósito de la gerontología contemporánea: la edad, el envejecimiento y el sujeto envejeciente (Alkema \& Alley, 2006).

Los enfoques en gerontología se enmarcan en dimensiones existenciales, colectivas y científicas. La dimensión existencial aborda la mitología, teatro y relatos populares, siendo relevante el estudio del tiempo privilegiado y los temas asociados a la eterna juventud. La dimensión colectiva observa el aumento del promedio de vida de los avances generados en la medicina, tecnología y sistemas de protección social, que han facilitado que más sujetos lleguen a la vejez (Laforest, 1991; Navarro, 2019). La dimensión científica incluye disciplinas gerontológicas y se divide en ramas o especialidades con los más variados nombres: gerontología biológica, gerontología social, psicogerontología, gerontología educativa, entre otras (Orellana, 2013).

En términos teóricos, la gerontología — con sus múltiples focos— puede diferenciarse en explicativa, comprensiva y crítica. Una gerontología explicativa tiende a cosificar el proceso de envejecimiento y establecer una relación distante entre el mundo científico y las personas mayores; es de orientación positivista e intenta demostrar que el conocimiento tiene como finalidad controlar la naturaleza y el progreso material (Piña, 2015; Vergara, 2014). Una gerontología comprensiva insta a profundizar en los significados socioculturales que van construyendo la realidad de las personas mayores y se basan en enfoques como la fenomenología y la hermenéutica (Piña-Morán et al., 2018). Una gerontología crítica genera un nexo con los movimientos sociales y no solo pretende comprender cómo se desarrolla la construcción social de la vejez, sino que además busca modificar tal visión mediante estrategias y políticas públicas. Se basa en enfoques que cuestionan el orden social y buscan aportar con una visión situada en una ruptura con la gerontología tradicional (Piña \& García, 2016; Piña \& Gómez, 2019; Yuni, 2019; Yuni \& Urbano, 2008).

El estudio del envejecimiento es interdisciplinario ya que incorpora un área de confluencia para diversas disciplinas y profesiones que forman parte de las ciencias de la salud, ciencias sociales, ciencias naturales y las áreas tecnológicas (Paredes, Lladó, \& Pérez, 2017). La formación gerontológica incluye conocimientos que se abocan a la investigación, intervención, atención y sistematización de experiencias con y junto a las personas mayores (Piña-Morán et al., 2018).

En las últimas décadas se ha observado un aumento importante de la investigación en el ámbito biomédico y vinculadas al cuidado (Pinquart \& Sörensen, 2003, 2007), el envejecimiento y funcionamiento cognitivo (Bopp \& Verhaeghen, 2005; Luchetti, Terracciano, Stephan, \& Sutin, 2016; Vanegas, Messa, Cardoza, Tovar, \& Ocampo, 2018), la depresión y soledad (Forsman, Schierenbeck, \& Wahlbeck, 2011; Kvelde et al., 2013; Masi, Chen, Hawkley, \& Cacioppo, 2011; Ong, Bert, Uchino, \& Wethington, 2016), y la enfermedad de Alzheimer (Ansari, Gul, \& Yaseen, 2006; Da Silva, Ramos, \& Carvalho, 2018; Shen, Nguyen, \& Hsu, 2019).

La producción de conocimientos en gerontología es un aporte primordial para la difusión y reflexión en este campo y su impacto en las políticas públicas con personas mayores (Delatin, Texeira, \& Sandroni, 2015). En ese sentido, es relevante considerar que la institucionalización del envejecimiento a nivel mundial ha implicado el desarrollo de dos Asambleas Mundiales, en Austria y España, que han destacado el aporte de la producción de conocimientos de la gerontología a nivel profesional y científico. En ambas asambleas se insta a los gobiernos a estimular la investigación, capacitación y educación en este ámbito (United Nations, 1983, 2002). Por otra parte, la Convención Interamericana sobre la Protección de los Derechos Humanos de las Personas Mayores, aprobada en junio del 2015 en la XLV Asamblea General Ordinaria de la Organización de Estados Americanos, plantea la necesidad de fortalecer la investigación y la formación académica profesional y técnica en geriatría y gerontología (OEA, 2015). 
En este escenario teórico y empírico que valora la construcción de conocimientos en disciplinas y profesiones asociadas al envejecimiento y vejez, surge este estudio orientado al análisis de los documentos de mayor impacto en gerontología, la evolución de las citaciones, y los autores, países y revistas con mayor productividad, a través de un análisis bibliométrico que permita aportar a la reflexión científica en este campo.

\section{Método}

El objetivo del presente estudio es caracterizar el desarrollo de la investigación en gerontología, identificando los estudios de mayor influencia en el área por medio de un análisis bibliométrico.

Los documentos de mayor impacto en un área de investigación son aquellos que marcan tendencia y son usados como referencia para estudios propios de una disciplina (Polanco-Carrasco, 2016). El concepto bibliométrico de citaciones clásicas fue establecido para designar aquellos trabajos más citados en un ámbito científico y su estructura (Garfield, 1977). Por su parte, el índice $h$ es un indicador bibliométrico que mide el factor de impacto en relación con el número de citas obtenidas en determinada producción científica (Hirsch, 2005). El concepto de $h$-clásico, utilizado en la presente investigación, se define como la identificación de las citaciones clásicas a través del índice h (Martínez, Herrera, López-Gijón, \& Herrera-Viedma, 2014). La base de datos Web of Science (WoS) ofrece una medida de $h$-clásico para cada área de investigación tomando en consideración el número de documentos y el impacto de éstos.

El procedimiento de búsqueda incluyó el descriptor "gerontology" para la búsqueda de artículos y revisiones en la colección principal de WoS de Thomson Reuters: Science Citation Index Expanded, Social Sciences Citation Index, Arts \& Humanities Citation Index y Emerging Sources Citation Index. No se establecieron restricciones de idioma. El tramo estudiado (que considera el año de inicio de los registros en WoS) es el comprendido entre 1975-2018 obteniéndose una base de datos con 3394 documentos.

El conjunto de datos obtenido fue procesado y corregido a través de una hoja de cálculo Excel. En paralelo, los archivos con los metadatos de los documentos fueron pre-procesados mediante el programa STICCI.eu (software diseñado para analizar conjuntos de datos bibliométricos) para unificar términos equivalentes. El archivo resultante fue importado a VOSviewer, versión 1.6.6., que es un software gratuito que permite construir y visualizar mapas bibliométricos (Van Leeuwen, 2004).

\section{Resultados}

En el campo de la investigación gerontológica el indicador $h$-clásico corresponde a los 94 documentos más citados en el área. El 75.5\% corresponde a artículos de investigación (71) y el $24.5 \%$ a revisiones y meta-análisis (23). La Tabla 1 resume las publicaciones, autores, revistas, citas y promedio anual de citaciones de aquellos textos clásicos que han marcado tendencia en la disciplina gerontológica en los últimos 44 años.

Los documentos clásicos han sido elaborados por autores de 26 países. Aquellos con mayor cantidad de publicaciones clásicas son Estados Unidos (70.2\%), seguido de Inglaterra (12.7\%), Canadá (10.6\%), Alemania $(8.5 \%)$ y España $(5.3 \%)$. La totalidad de estas publicaciones se realizó en idioma inglés.

Considerando las definiciones WoS los textos de mayor impacto son clasificados en las categorías: "gerontology" ("gerontología") que concentra el 41.4\% de las publicaciones clásicas. En segundo lugar, aparece "geriatrics gerontology" ("geriatría gerontológica") con un 25.5\%, "psychology multidisciplinary" ("psicología multidisciplinaria") (7.4\%), "multidisciplinary sciences" ("ciencias multidisciplinarias") (6.3\%) y, finalmente, "clinical neurology" ("neurología clínica"), "psychology” ("psicología”) y "public environmental occupational health" ("salud ocupacional pública ambiental") con un $5.3 \%$ respectivamente.

Las áreas de investigación gerontológica más estudiadas, corresponden en un $54.2 \%$ a "geriatrics gerontology" ("geriatría gerontológica"), 10.6\% a "psychology" ("psicología”) y 7.4\% a "neurociences neurology" ("neurociencias neurología"). 


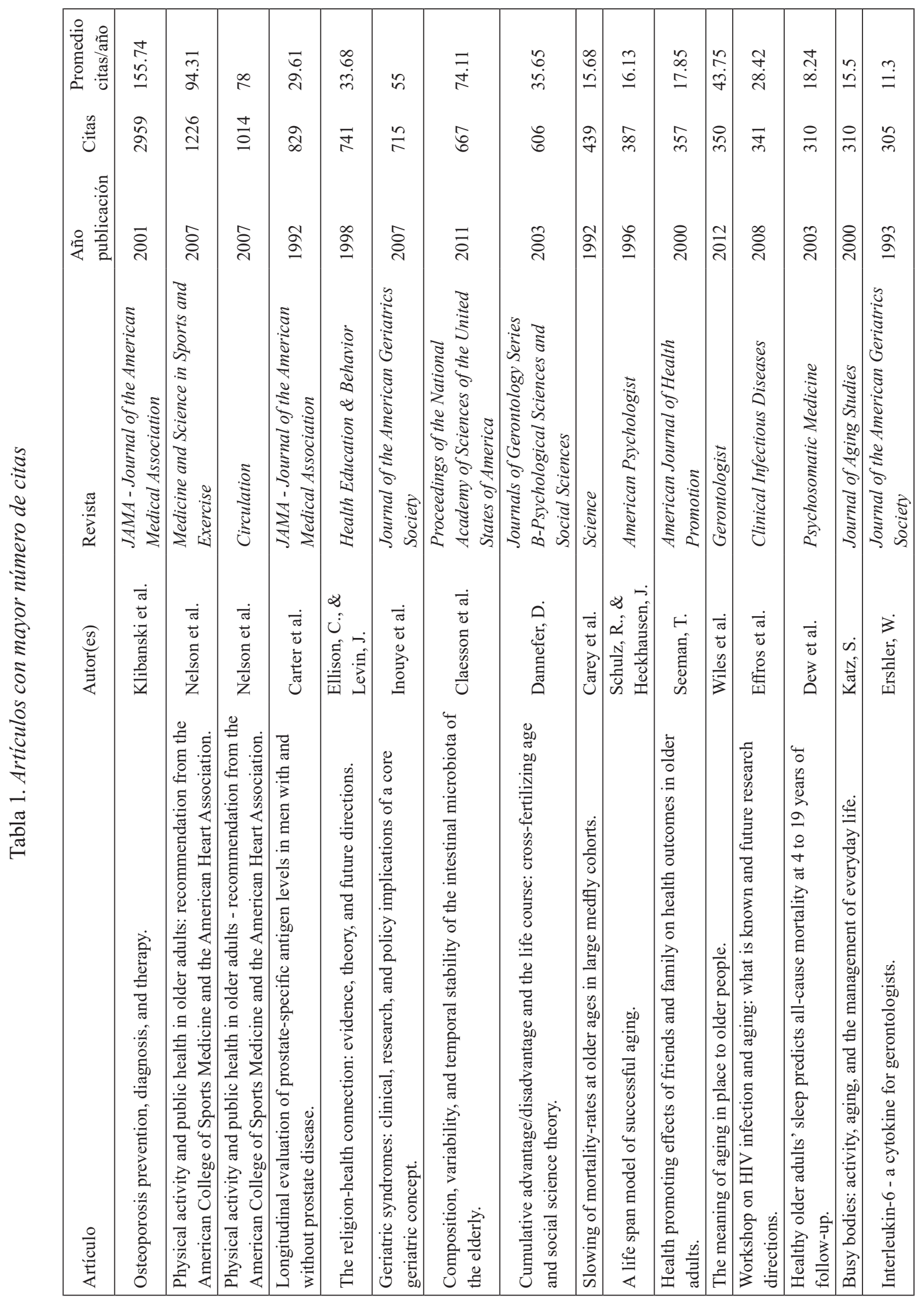


Polanco-CarRasco \& María Gladys Olivo-Viana

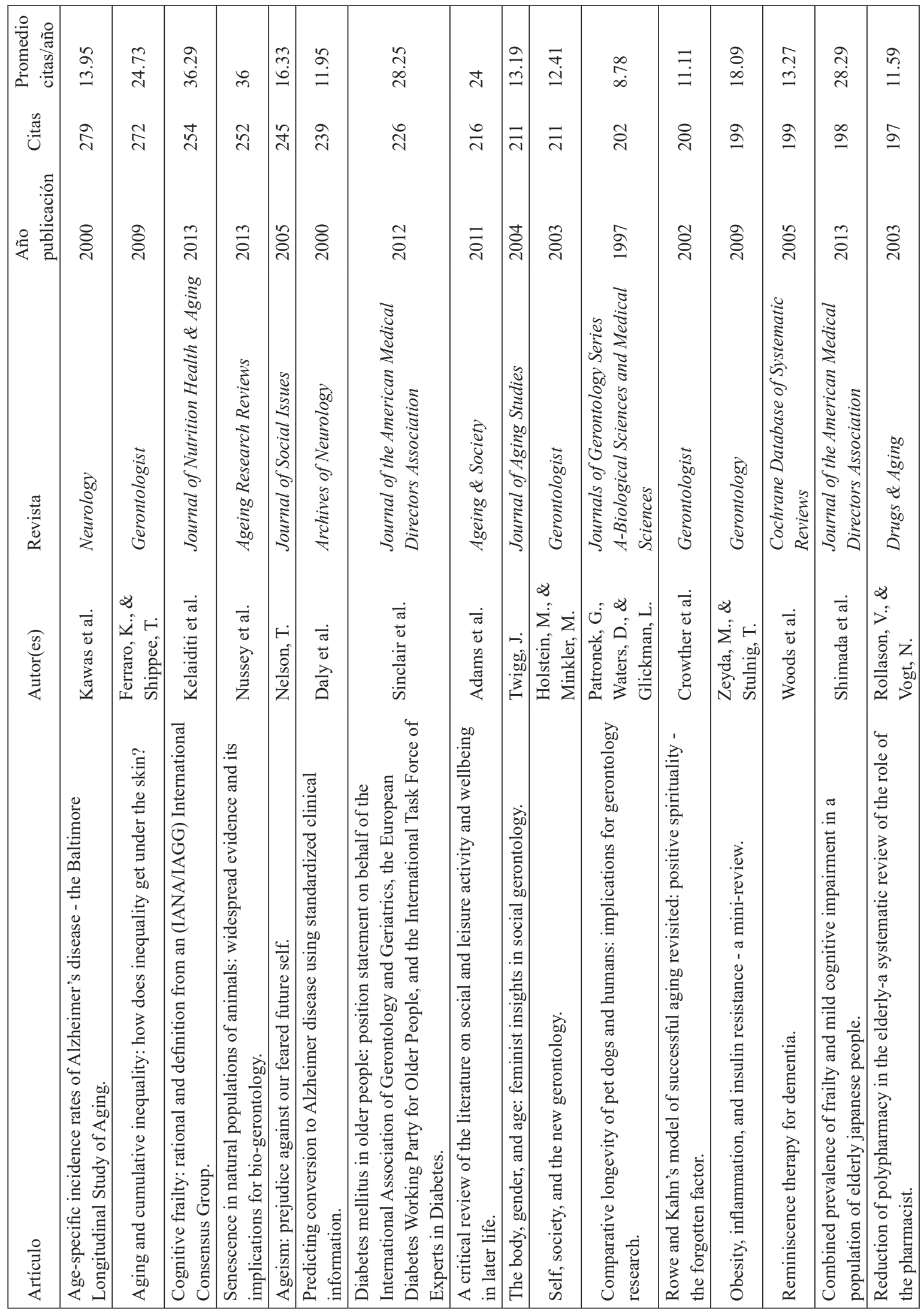


Gerontología: UN MIRADA BIBLIOMÉTRICA

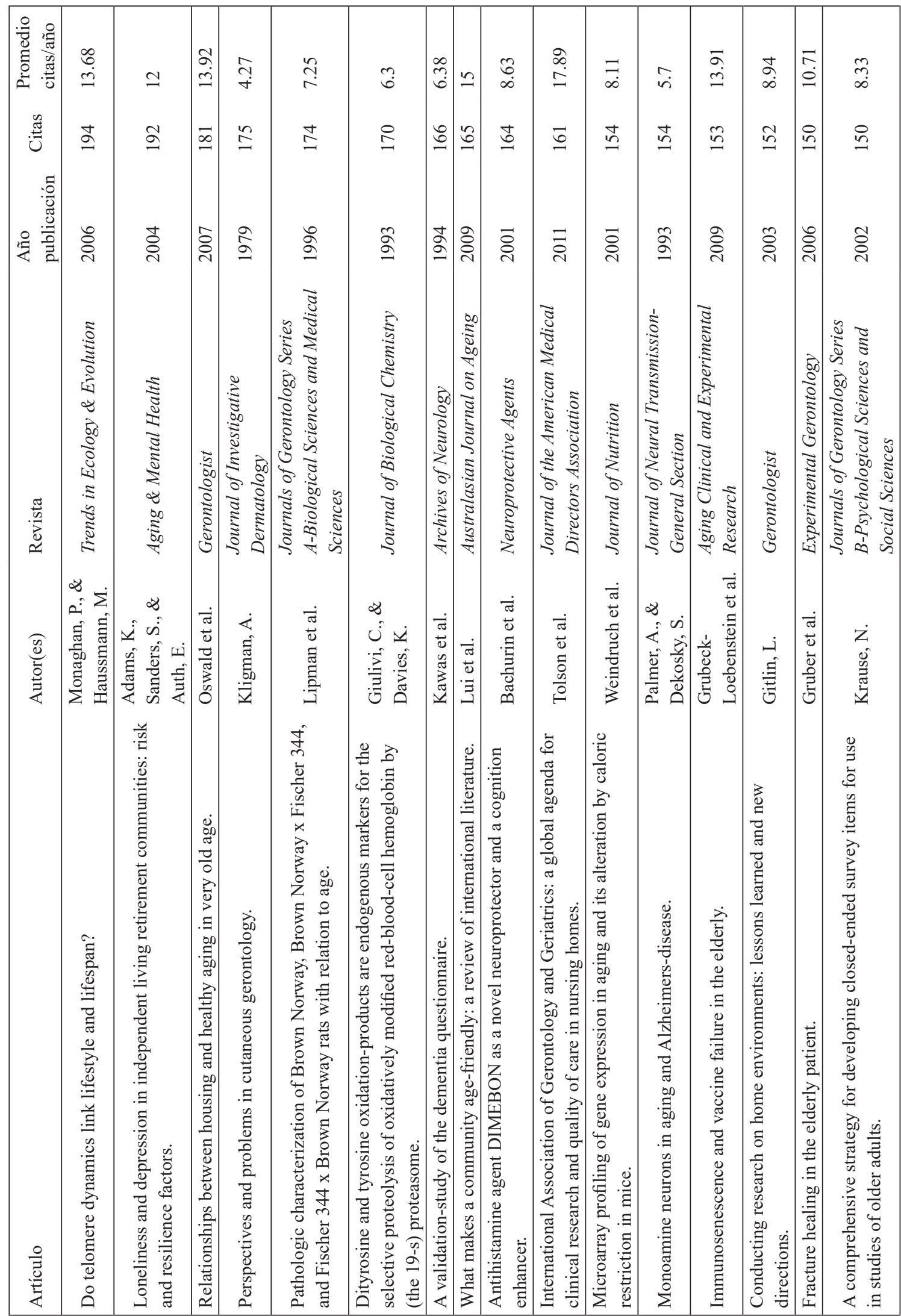


Polanco-Carrasco \& María Gladys Olivo-Viana

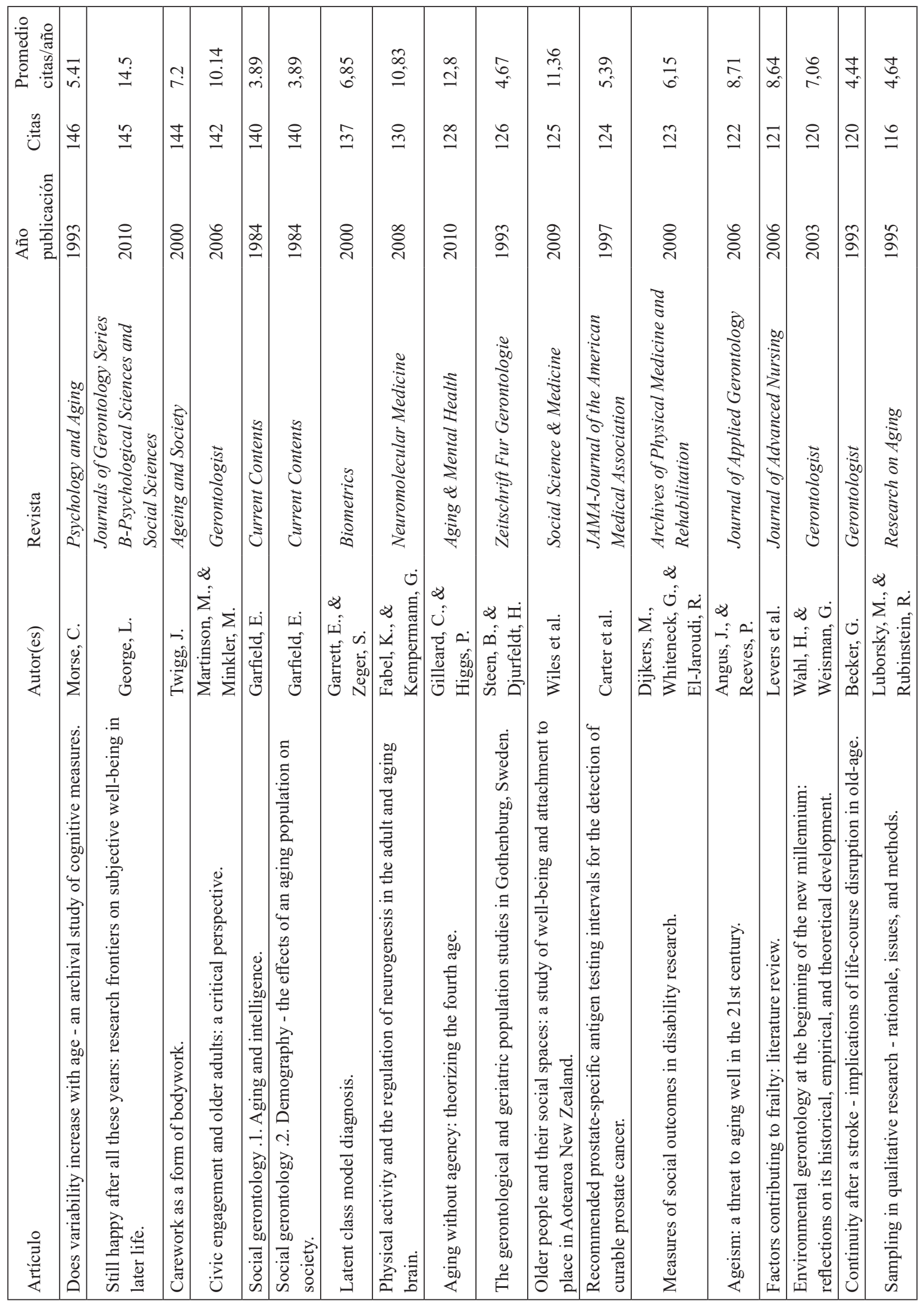


GERONTOLOGÍA: UN MIRADA BIBLIOMÉTRICA

\begin{tabular}{|c|c|c|c|c|c|c|c|c|c|c|c|c|c|c|c|c|}
\hline 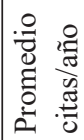 & 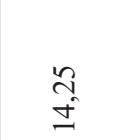 & $\stackrel{\widehat{N}}{\infty}$ & $\stackrel{\cong}{\sim}$ & $\begin{array}{l}0 \\
\text { in }\end{array}$ & $\begin{array}{l}\stackrel{0}{2} \\
\text { sn }\end{array}$ & $\begin{array}{l}\infty \\
\text { in }\end{array}$ & $\begin{array}{l}\bar{m} \\
f\end{array}$ & 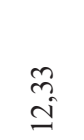 & $\begin{array}{l}\hat{0} \\
0 \\
0\end{array}$ & \begin{tabular}{l}
\multirow{n}{n}{} \\
$\infty$
\end{tabular} & $\frac{\Omega}{n}$ & $\begin{array}{l}\stackrel{\approx}{\alpha} \\
\dot{\sigma}\end{array}$ & $\stackrel{n}{n}$ & 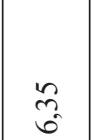 & $\sqrt{n}$ & r \\
\hline$\stackrel{\mathscr{ٌ}}{\tilde{U}}$ & $\stackrel{\Xi}{\Xi}$ & $\stackrel{\Xi}{\Xi}$ & $\Xi$ & $\stackrel{m}{\Xi}$ & $\stackrel{\varrho}{\exists}$ & $\cong$ & $\Xi$ & $\Xi$ & $\Xi$ & $\Xi$ & $\Xi$ & $\stackrel{\circ}{ }$ & 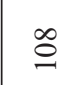 & $\stackrel{\infty}{\circ}$ & $\stackrel{0}{\circ}$ & $\stackrel{2}{\varrho}$ \\
\hline 要皇 & $\stackrel{\sim}{\stackrel{\sim}{\sim}}$ & ڤ్రి & 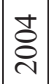 & $\stackrel{n}{\stackrel{\sim}{\sim}}$ & $\overline{\vec{d}}$ & ర్రి & वे & $\overrightarrow{\vec{d}}$ & ஓे & ڤ్ & \&્స & $\begin{array}{l}\infty \\
\sigma \\
\sigma\end{array}$ & 공 & ڤ్రి & & రి \\
\hline 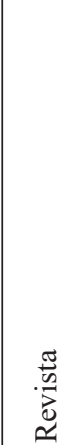 & 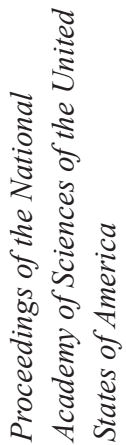 & 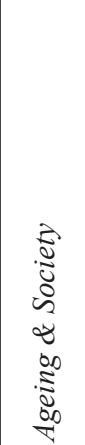 & 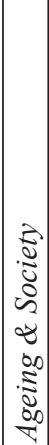 & 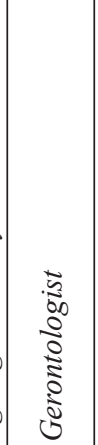 & 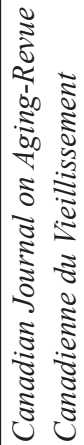 & 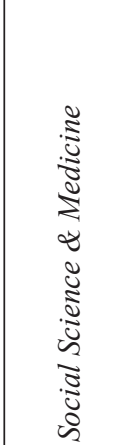 & 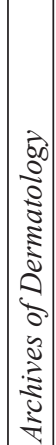 & 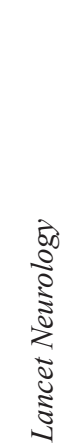 & $\begin{array}{l}\widetilde{\Xi} \\
0 \\
.0 \\
.0 \\
0 \\
0\end{array}$ & 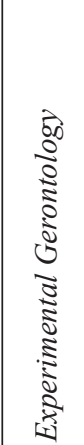 & 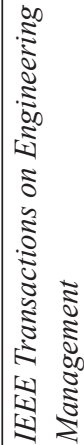 & 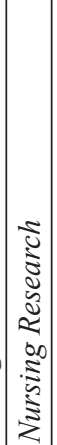 & 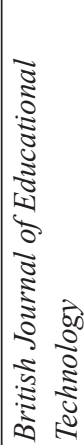 & 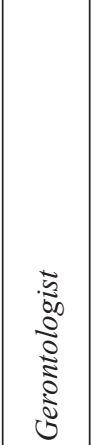 & 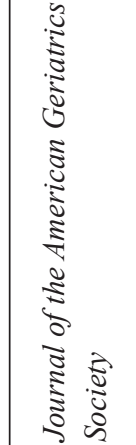 & $\begin{array}{l}\overline{5} \\
\text { s. } \\
0 \\
0 \\
0 \\
0 \\
0 \\
0 \\
0\end{array}$ \\
\hline 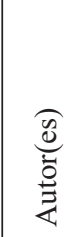 & 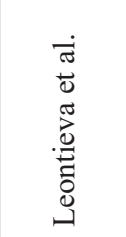 & 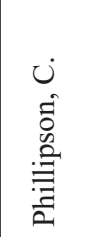 & 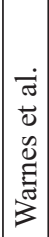 & 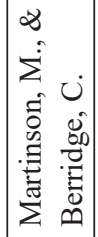 & 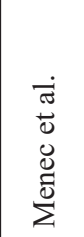 & 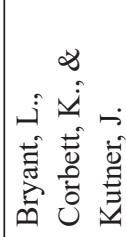 & $\begin{array}{c}\dot{\Sigma} \\
\dot{\Sigma} \\
\dot{s} \\
\dot{s} \\
3\end{array}$ & $\begin{array}{l}\dot{\sigma} \\
\tilde{0} \\
\tilde{0} \\
\tilde{\sigma} \\
\tilde{0} \\
0\end{array}$ & 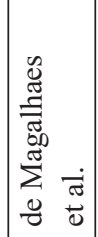 & 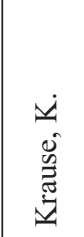 & 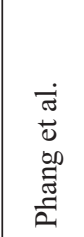 & $\mid \begin{array}{l}> \\
\dot{\Xi} \\
\dot{0}\end{array}$ & 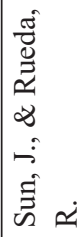 & 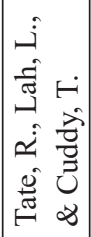 & 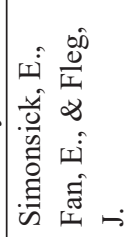 & 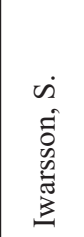 \\
\hline 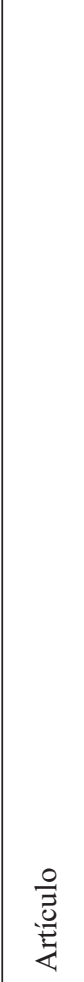 & 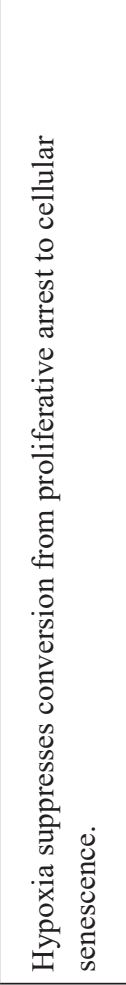 & 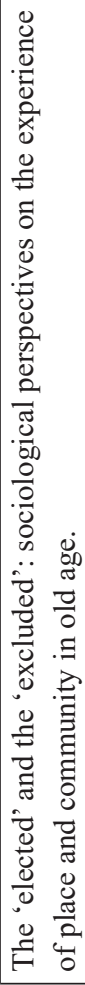 & 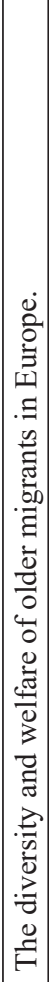 & 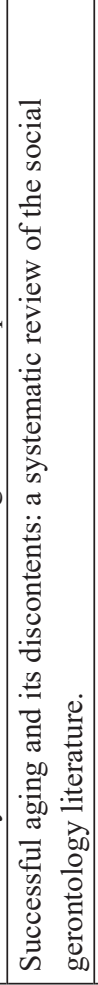 & 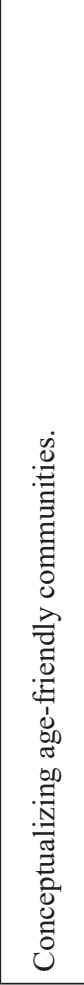 & 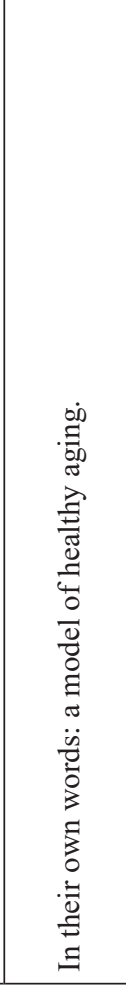 & 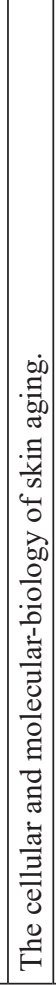 & 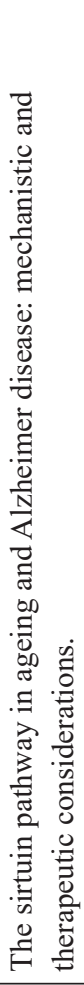 & 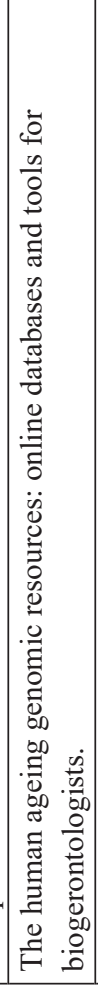 & 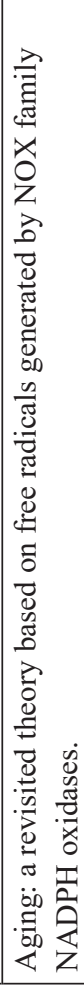 & 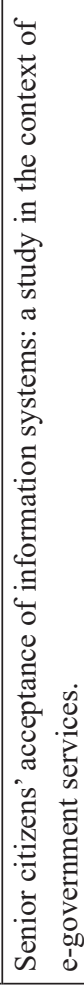 & 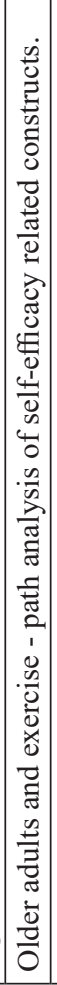 & 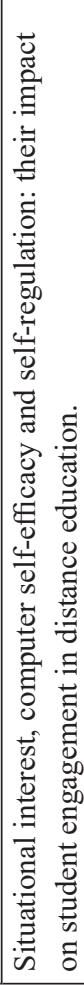 & 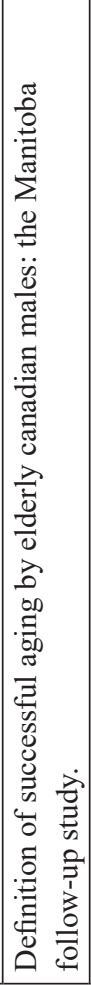 & 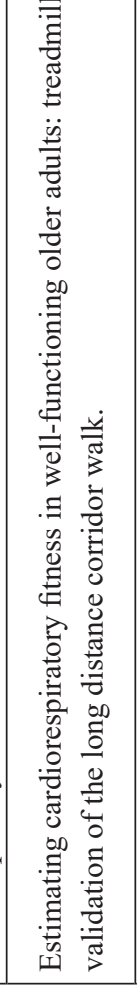 & 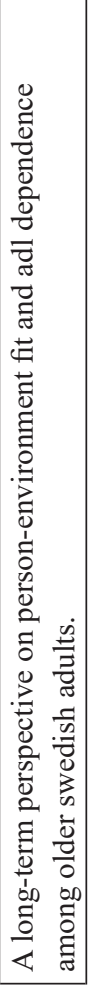 \\
\hline
\end{tabular}


Polanco-Carrasco \& María Gladys Olivo-Viana

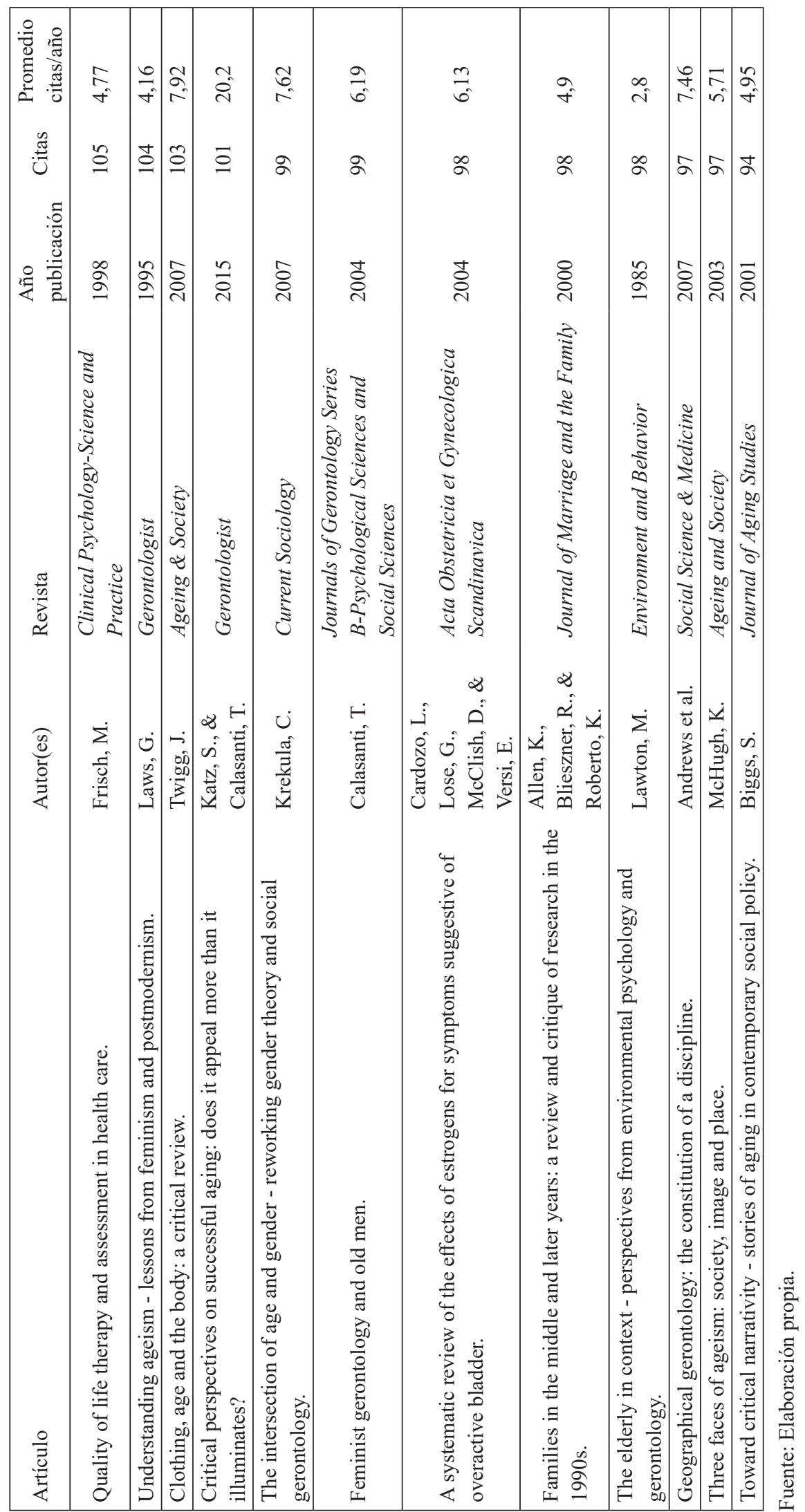


Un análisis longitudinal da cuenta de la producción de los documentos clásicos en gerontología durante el periodo estudiado. En la Figura 1 se observa que los 94 textos se distribuyen de manera irregular destacando el año 2007 con el mayor número de textos clásicos publicados (9). El más antiguo corresponde a "Perspectives and problems in cutaneous gerontology" que aborda el envejecimiento fisiológico y patológico de la piel y su impacto en la calidad de vida (Kligman, 1979).

Los últimos dos textos clásicos en la disciplina fueron publicados el año 2015, en la revista The Gerontologist, donde analizan los alcances y limitaciones del modelo de envejecimiento exitoso desde la gerontología social y gerontología crítica (Katz \& Calasanti, 2015; Martinson \& Berridge, 2014).

Figura 1. Número de publicaciones por año.

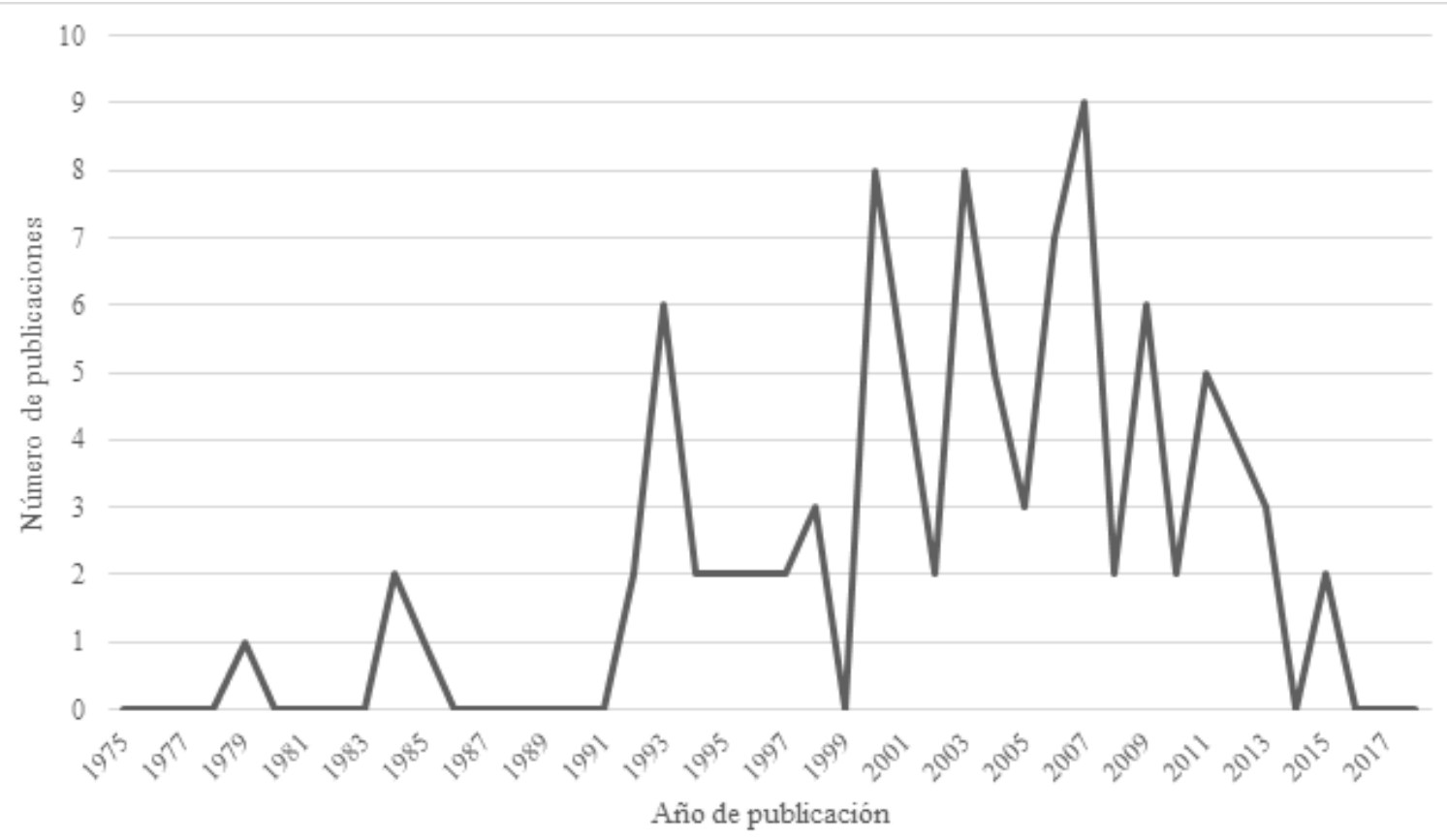

Fuente: Elaboración propia.

Al considerar el nivel de impacto de los textos clásicos (ver Figura 2), el periodo 1997-2007 destaca como el tramo de publicaciones con mayor cantidad de citas en el ámbito gerontológico concentrando el $62.57 \%$ de ellas. El 2007 y 2001 fueron los años donde se publicaron los documentos de mayor impacto. 
Figura 2. Citaciones de artículos clásicos entre 1975 y 2018.

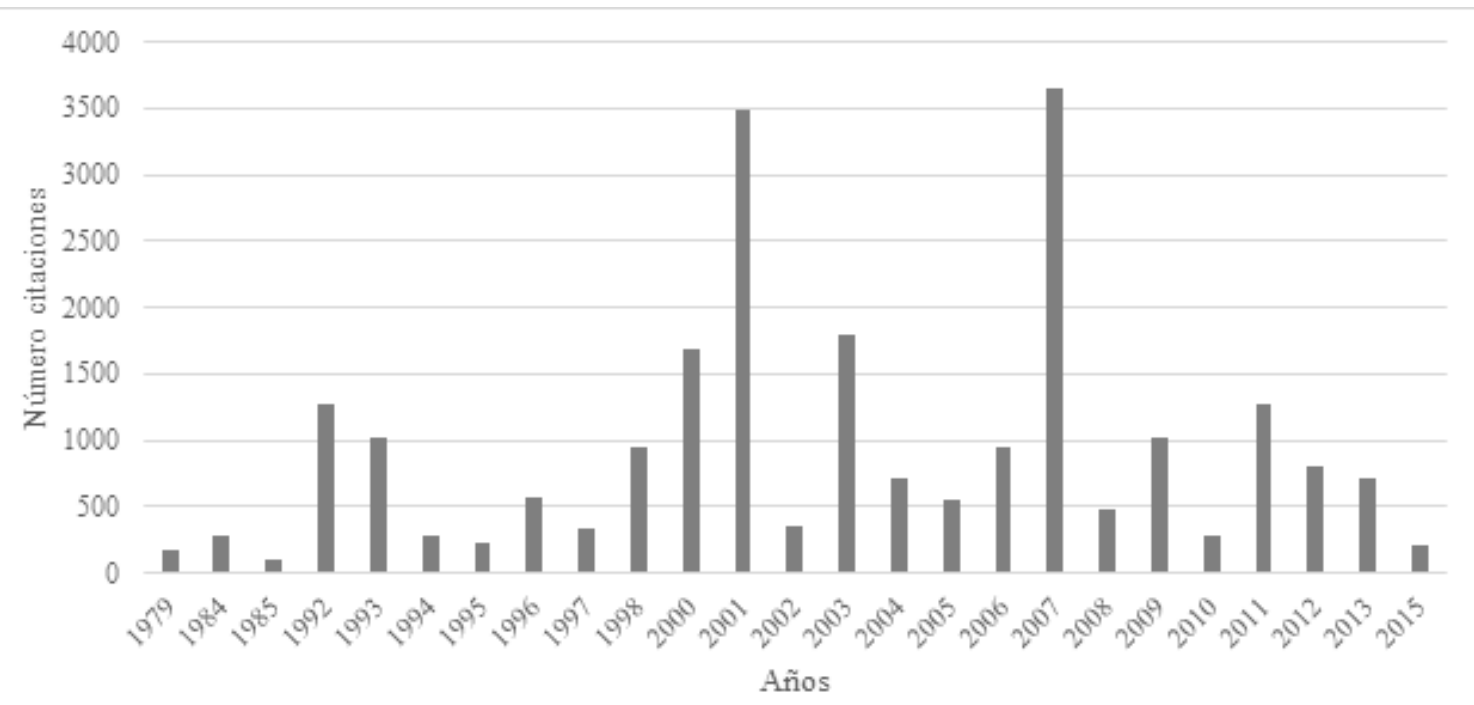

Fuente: Elaboración propia.

Durante el año 2007, donde fueron publicados los textos con mayor volumen de citas (15.8\%), las temáticas tratadas en sus artículos se relacionan con: a) la evidencia respecto a los tipos y cantidad de actividad física necesaria para mejorar y mantener la salud en los adultos mayores (Nelson et al., 2007), b) los criterios para definir los síndromes geriátricos, identificando que la edad mayor, el deterioro cognitivo y funcional inicial, así como la alteración de la movilidad, son factores de riesgo compartidos para las úlceras por presión, incontinencia, caídas, deterioro funcional y delirio (Inouye, Studenski, Tinetti, \& Kuchel, 2007), c) la relación entre la vivienda y los aspectos del envejecimiento saludable, como la independencia en las actividades diarias y el bienestar subjetivo, comparando cinco países europeos: Suecia, Alemania, Gran Bretaña, Hungría y Letonia (Oswald et al., 2007), d) el impacto de los cambios sociales, culturales y económicos derivados de la globalización y su efecto en las personas mayores debido a las nuevas divisiones sociales, entre quienes pueden elegir donde residir en concordancia con su historia de vida, y aquellos que viven el rechazo o marginación de su localidad (Phillipson, 2007), e) el efecto de los radicales libres en el envejecimiento y el rol de cierto tipo de enzimas vinculadas a este proceso (Krause, 2007), f) el significado de la vestimenta de los ancianos en la construcción y experiencia de la vejez en relación con el cuerpo, la identidad y la agencia personal (Twigg, 2007), g) la posición de las mujeres mayores, en la teoría de género y gerontología social, desde la intersección entre género y edad (Krekula, 2007), y h) la evolución de este campo disciplinar, entre los años 1995-2006, en la temática de salud de la población anciana (Andrews, Cutchin, McCracken, Phillips, \& Wiles, 2007).

El año 2001 fue publicado el segundo grupo de textos más relevantes en gerontología con un $15 \%$ del total de citaciones en los 44 años estudiados. En ellos se abordan temáticas tales como: a) los resultados beneficiosos del fármaco antihistamínico Dimebon en la terapia para el Alzheimer (Bachurin et al., 2001), b) el estudio en el área de la gerontología biológica respecto al impacto de la restricción calórica en roedores y su efecto en el proceso de envejecimiento (Weindruch, Kayo, Lee, \& Prolla, 2001), c) identificar, a través de la teoría fundamentada, los factores que discriminan entre un envejecimiento más y menos saludable desde la perspectiva de las personas mayores (Bryant, Corbett, \& Kutner, 2001), y d) una evaluación desde una narrativa crítica de las políticas de envejecimiento positivo de América del Norte y parte de Europa (Biggs, 2001).

Los documentos clásicos en gerontología son divulgados en 57 revistas distribuidas en 9 países. Estados Unidos concentra el $56.1 \%$ de las publicaciones y además el mayor volumen de citas como se observa en la Tabla 2 . 
Tabla 2. Revistas con mayor número de citas.

\begin{tabular}{|c|c|c|c|}
\hline Revista & Citaciones & $\%$ & País \\
\hline Journal of the American Medical Association & 3912 & 17 & EEUU \\
\hline The Gerontologist & 2279 & 10 & EEUU \\
\hline Medicine and Science in Sports and Exercise & 1226 & 5.3 & EEUU \\
\hline Journal of the American Geriatrics Society & 1126 & 4.9 & EEUU \\
\hline Circulation & 1014 & 4.4 & EEUU \\
\hline Journals of Gerontology Series B-Psychological Sciences and Social Sciences & 1000 & 4.3 & EEUU \\
\hline $\begin{array}{l}\text { Proceedings of the National Academy of Sciences of the United States of } \\
\text { America }\end{array}$ & 781 & 3.8 & EEUU \\
\hline Health Education \& Behavior & 741 & 3.2 & EEUU \\
\hline Journal of Aging Studies & 615 & 2.6 & UK \\
\hline Journal of the American Medical Directors Association & 585 & 2.5 & EEUU \\
\hline
\end{tabular}

Fuente: Elaboración propia.

Los documentos clásicos corresponden a 26 países, liderado por Estados Unidos (51.8\%), seguido de Canadá e Inglaterra (5.8 y $4.4 \%$, respectivamente).

Los textos más citados en gerontología corresponden a 359 autores. Como señala la Tabla 3 aquellos con mayor impacto concentran el $28 \%$ (40.714) del total de las citaciones (144.330). El texto clásico más citado en el área gerontológica corresponde a la revisión "Osteoporosis prevention, diagnosis, and therapy" que concentra el $12.8 \%$ de las citas (2.959). El documento aporta la sistematización de una conferencia que reunió a 32 expertos en este campo que expusieron respecto a la evidencia científica en relación a la osteoporosis (Klibanski et al., 2001). Steven Blair es el autor que lidera el total de citaciones del periodo estudiado. Sus artículos corresponden al ámbito de la medicina y ciencias de la salud, y están vinculados principalmente a la actividad física (Blair et al., 1995; Blair \& Haskell, 2006; Huang et al., 1998; Klibasnki et al., 2001; Nelson et al., 2007; Sui et al., 2007).

Tabla 3. Autores con mayor número de citas.

\begin{tabular}{lll}
\hline Autor & $\mathrm{N}^{\circ}$ Documentos & Citaciones \\
\hline Blair, S. & 3 & 5194 \\
Adams-Campbell, L. & 1 & 2960 \\
Bassford, T. & 1 & 2960 \\
Boden, S.D. & 1 & 2960 \\
Dickersin, K. & 1 & 2960 \\
Gifford, D.R. & 1 & 2960 \\
Glasse, L. & 1 & 2960 \\
Goldring, S.R. & 1 & 2960 \\
Hruska, K. & 1 & 2960 \\
Johnson, S.R. & 1 & 2960 \\
Klibanski, A. & 1 & 2960 \\
Mccauley, L.K. & 1 & 2960 \\
Russell, W.E. & 1 & 2960 \\
\hline
\end{tabular}

Fuente: Elaboración propia. 
$\mathrm{Al}$ analizar la agrupación de autores de textos clásicos se aprecia que el máximo de documentos desarrollados mutuamente no supera los tres artículos. Para efectos de un análisis más específico se consideró la co-autoría como al menos un artículo desarrollado en conjunto. Del total de autores sólo el 13.6\% (49) cumplen con ese criterio conformando tres grandes conglomerados concentrados entre los años 2011-2013 (ver Figura 3). Los investigadores Yves Rolland, Sandrine Andrieu y Antoni Salvà, funcionan como nexo entre el conglomerado 2013 y 2011, y todos estos autores presentan publicaciones en el ámbito biomédico. El primero, con textos vinculados a la caquexia, sarcopenia y fragilidad (Rolland, Dupuy, van Kan, Gillette, \& Vellas, 2011; Rolland, van Kan, Gillette-Guyonnet, \& Vellas, 2011); El segundo, con publicaciones relacionadas a la enfermedad de Alzheimer (Hampel et al., 2011; Vellas et al., 2012); Y el tercer autor, vinculado a temas nutricionales en ancianos y de percepción de salud (Roqué, Salvà, Bolíbar, \& Rivero, 2012; Salvà, 2012).

Figura 3. Conglomerados de co-autoría de autores clásicos.

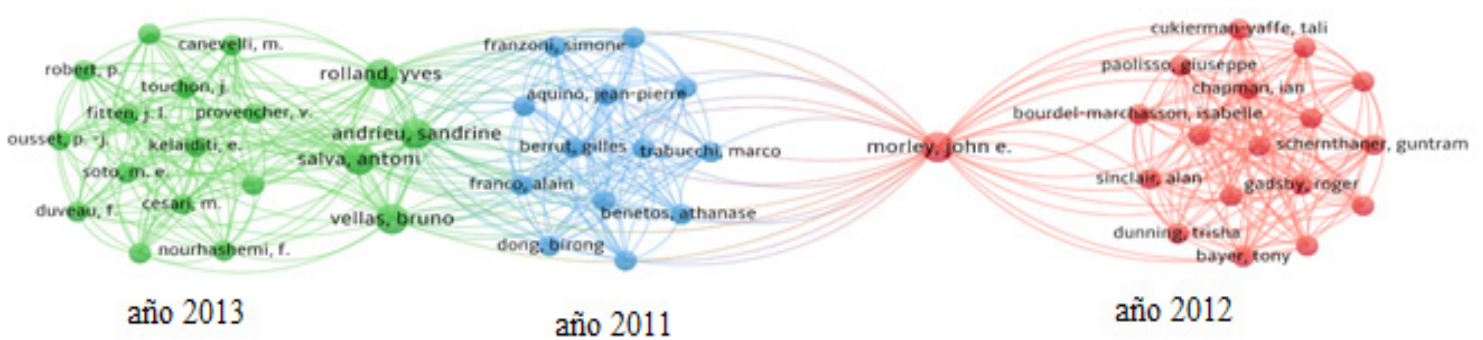

Fuente: Elaboración propia.

John Morley es el autor que conecta a los investigadores que publican en co-autoría entre los años 2011 y 2012, y más débilmente con aquellos del año 2013. Los temas abordados en sus trabajos se vinculan a la fragilidad clínica, la pérdida de masa y función muscular esquelética asociada a la edad y las consecuencias para el desarrollo de la fragilidad y la discapacidad (Morley et al., 2013; Fielding et al., 2011; Woo, Leung, \& Morley, 2012).

Al analizar la relación entre los textos clásicos, de acuerdo a las referencias bibliográficas compartidas, se identifican los subcampos de investigación gerontológica que comparten una base conceptual común. Se definió como criterio para este análisis el presentar una referencia compartida. De los 94 textos clásicos, 69 cumplen con este criterio conformando ocho conglomerados. El primer agrupamiento aborda temáticas de salud, religión, actividad social y bienestar (Adams, Leibbrandt, \& Moon, 2011; Ellison \& Levin, 1998; Seeman, 2000). El segundo conglomerado, versa respecto a patologías médicas como la enfermedad próstatica en hombres mayores y patologías del sistema digestivo (Carter et al., 1992; Carter, Epstein, Chan, Fozard, \& Pearson, 1997; Claesson et al., 2011). El tercer conglomerado aborda temáticas desde la gerontología crítica, como el cuestionamiento a los modelos de envejecimiento positivo y el exitoso (Biggs, 2001; Holstein \& Minkler, 2003), así como la discriminación y fragmentación de la vejez (Gilleard \& Higgs, 2010). El cuarto conglomerado, trata de la relación entre la edad y la teoría de ventaja y desventaja acumulativa (Dannefer, 2003), la relación entre la edad y la mortalidad (Carey, Liedo, Orozco, \& Vaupel, 1992), y la incorporación de aspectos no normativos y de la trayectoria de vida al modelo de envejecimiento exitoso (Schulz \& Heckhausen, 1996). El quinto conglomerado trata de la importancia del contexto donde se envejece, a través de los trabajos respecto al significado y apego para las personas adultas y adultas mayores de envejecer en su lugar de residencia (Wiles, Leibing, Guberman, Reeve, \& Allen, 2012; Wiles et al., 2009) y la calidad de la atención para la salud en ancianos institucionalizados (Tolson et al., 2011). El sexto conglomerado se vincula a la gerontología ambiental respecto al envejecimiento, la vivienda y la relación de las personas mayores y su entorno (Gitlin, 2003; Oswald et al., 2007; Wahl \& Weisman, 2003). El séptimo conglomerado se focaliza en las comunidades para la vejez (Conn, 1998; Lui, Everingham, Warburton, Cuthill, \& Bartlett, 2009; Menec, Means, Keating, Parkhurst, \& Eales, 2011). Y, 
finalmente, el octavo conglomerado aborda temas — desde la gerontología social y crítica — como la relación entre el prejuicio hacia las personas mayores y el buen envejecimiento (Angus \& Reeve, 2006) y críticas al modelo de envejecimiento exitoso (Katz \& Calasanti, 2015; Martinson \& Berridge, 2014).

\section{Conclusiones}

El objetivo del presente estudio fue caracterizar los textos de mayor impacto en el ámbito gerontológico en la colección principal de la base de datos Web of Science en el periodo comprendido entre los años 1975-2018. Se identificaron 94 publicaciones clásicas en el área que se describieron de acuerdo al tipo de documentos, países, áreas de investigación, evolución longitudinal, análisis de co-autoría y acoplamiento de textos clásicos.

La mayoría de los textos corresponden a artículos y el área de investigación predominante es la "geriatría gerontológica". Estados Unidos es el país que concentra el mayor número de publicaciones, citas y fuentes de difusión científica a nivel gerontológico con una hegemonía esperable del idioma inglés en la producción científica identificada.

La producción de textos clásicos es irregular y los más relevantes se concentran en la década 1997-2007. El porcentaje de co-autoría de los autores de textos clásicos es baja y se concentra entre los años 2011-2013.

$\mathrm{Al}$ analizar la evolución de los textos más citados, comparando el primero y los últimos, se aprecia una apertura en los temas de estudio desde un inicial foco en lo biomédico hasta ulterior incorporación de la gerontología social y crítica en el cuestionamiento a ciertos modelos de envejecimiento (Curcio, 2014; Piña \& Gómez, 2019).

Se identifican subdisciplinas en el campo gerontológico a través de las referencias bibliográficas compartidas, reafirmándose la interdisciplinariedad en gerontología, ya que su producción de conocimientos abarca áreas de la salud, la gerontología crítica, los modelos en envejecimiento, la calidad de vida, la gerontología ambiental y los enfoques de comunidad.

Son limitaciones del presente estudio la utilización de un único indicador bibliométrico ( $h$-clásico) para determinar las publicaciones más relevantes en el ámbito gerontológico, entendiendo que el prestigio o influencia de un cúmulo de investigaciones pueden ser abordadas por medio de otros indicadores adicionales (Polanco-Carrasco, 2016), quedando como un importante desafío para nuevos estudios similares. Por otra parte, otra limitante en esta investigación - a considerar en un futuro análisis de la producción científica en gerontología - implica involucrar otros aspectos como la co-ocurrencias de palabras claves, la relación entre instituciones que publican y financian las investigaciones de mayor impacto y la red de colaboración entre países. Otro desafío pendiente implica analizar la producción de conocimientos en América Latina y el Caribe (Polanco-Carrasco, 2017) que fundamentan las matrices de pensamiento epistemológicas, teóricas y metodológicas en gerontología — contemplando la revisión de artículos con mayor impacto y tendencia - en contextos diferentes al norteamericano o europeo.

\section{Referencias}

Adams, K., Leibbrandt, S., \& Moon, H. (2011). A critical review of the literature on social and leisure activity and wellbeing in later life. Ageing and Society, 31, 683-712. https://doi.org/10.1017/S0144686X10001091

Alkema, G. E., \& Alley, D. E. (2006). Gerontology's future: an integrative model for disciplinary Advancement. The Gerontologist, 46, 574-582. https://doi.org/10.1093/geront/46.5.574

Andrews, G. J., Cutchin, M., McCracken, K., Phillips, D. R., \& Wiles, J. (2007). Geographical Gerontology: The constitution of a discipline. Social Science \& Medicine, 65, 151-168. https://doi.org/10.1016/j.socscimed.2007.02.047

Angus, J., \& Reeve, P. (2006). Ageism: a threat to "aging well" in the 21st century. Journal of Applied Gerontology, 25, 137-152. https:// doi.org/10.1177/0733464805285745

Ansari, M. A., Gul, S., \& Yaseen, M. (2006). Alzheimer's disease: a bibliometric study. Trends in Information Management, 2, $130-140$.

Bachurin, S., Bukatina, E., Lermontova, N., Tkachenko, S., Afanasiev, A., Grigoriev, V., \& Zefirov, N. (2001). Antihistamine agent Dimebon as a novel neuroprotector and a cognition enhancer. Annals of the New York Academy of Sciences, 939, 425-435. https:// doi.org/10.1111/j.1749-6632.2001.tb03654.x

Biggs, S. (2001). Toward critical narrativity: stories of aging in contemporary social policy. Journal of Aging Studies, 15, 303-316. https://doi.org/10.1016/S0890-4065(01)00025-1 
Blair, S. N., Kohl, H. W., Barlow, C. E., Paffenbarger, R. S., Gibbons, L. W., \& Macera, C. A. (1995). Changes in physical fitness and all-cause mortality: a prospective study of healthy and unhealthy men. JAMA, 273, 1093-1098.

Blair, S. N., \& Haskell, W. L. (2006). Objectively measured physical activity and mortality in older adults. JAMA, 296, 216-218. https:// doi.org/10.1001/jama.296.2.216

Bopp, K. L., \& Verhaeghen, P. (2005). Aging and verbal memory span: a meta-analysis. Journals of Gerontology - Series B Psychological Sciences and Social Sciences, 60, 223-233. http://doi.org/10.1093/geronb/60.5.P223

Bryant, L. L., Corbett, K. K., \& Kutner, J. S. (2001). In their own words: a model of healthy aging. Social Science \& Medicine, 53, 927941. https://doi.org/10.1016/S0277-9536(00)00392-0

Carey, J., Liedo, P., Orozco, D., \& Vaupel, J. W. (1992). Slowing of mortality rates at older ages in large medfly cohorts. Science, 258, 457-461. https://doi.org/10.1126/science.1411540

Carter, H. B., Epstein, J. I., Chan, D. W., Fozard, J. L., \& Pearson, J. D. (1997). Recommended prostate-specific antigen testing intervals for the detection of curable prostate cancer. JAMA, 277, 1456-1460. https://doi.org/10.1001/jama.1997.03540420052029

Carter, H. B., Pearson, J. D., Metter, E. J., Brant, L. J., Chan, D. W., Andres, R., ... \& Walsh, P. C. (1992). Longitudinal evaluation of prostate-specific antigen levels in men with and without prostate disease. JAMA, 267, 2215-2220. https://doi.org/10.1001/ jama.1992.03480160073037

Claesson, M., Cusack, S., O’Sullivan, O., Greene-Diniz, R., de Weerd, H., Flannery, E., ... O’Toole, P. (2011). Composition, variability, and temporal stability of the intestinal microbiota of the elderly. Proceedings of the National Academy of Sciences, 108, 4586-4591. https://doi.org/10.1073/pnas.1000097107

Conn, V. S. (1998). Older adults and exercise: path analysis of self-efficacy related constructs. Nursing Research, 47, 180-189. https:// doi.org/10.1097/00006199-199805000-00009

Curcio, C. L., (2014). Envejecimiento exitoso: consideraciones críticas. Revista de la Asociación Colombiana de Geriatría y Gerontología, 28, 1955-1977.

Dannefer, D. (2003). Cumulative advantage/disadvantage and the life course: cross-fertilizing age and social science theory. The Journals of Gerontology: Series B, 58, S327-S337. https://doi.org/10.1093/geronb/58.6.S327

Da Silva, C., Ramos, V., \& Carvalho, P. (2018). Incremento de los cambios cognitivos en pacientes con enfermedad de Alzheimer inicial debido a depresión. Cuadernos de Neuropsicología - Panamerican Journal of Neuropsychology, 12, 42-51. https://doi.org/10.7714/ cnps/12.1.203

Delatin, D., Texeira, M. J., \& Sandroni, L. (2015). De la investigación a la política pública: producción y circulación de conocimiento científico. Revista Nómadas, 42, 150-165.

Ellison, C. G., \& Levin, J. S. (1998). The religion-health connection: evidence, theory, and future directions. Health Education \& Behavior, 25, 700-720. https://doi.org/10.1177/109019819802500603

Fielding, R. A., Vellas, B., Evans, W. J., Bhasin, S., Morley, J. E., Newman, A. B., ... \& Cederholm, T. (2011). Sarcopenia: an undiagnosed condition in older adults. Current consensus definition: prevalence, etiology, and consequences. International working group on sarcopenia. Journal of the American Medical Directors Association, 12, 249-256. https://doi.org/10.1016/j.jamda.2011.01.003

Forsman, A. K., Schierenbeck, I., \& Wahlbeck, K. (2011). Psychosocial interventions for the prevention of depression in older adults: systematic review and meta-analysis. Journal of Aging and Health, 23, 387-416. http://doi.org/10.1177/0898264310378041

Garfield, E. (1977). Citation indexing for studying science. Nature, 227, 669-671.

Gilleard, C., \& Higgs, P. (2010). Aging without agency: theorizing the fourth age. Aging \& Mental Health, 14, 121-128. https://doi. org/10.1080/13607860903228762

Gitlin, L. (2003). Conducting research on home environments: lessons learned and new directions. The Gerontologist, 43, 628-637. https://doi.org/10.1093/geront/43.5.628

Hampel, H., Wilcock, G., Andrieu, S., Aisen, P., Blennow, K., Broich, K., ... \& Lovestone, S. (2011). Biomarkers for Alzheimer's disease therapeutic trials. Progress in Neurobiology, 95, 579-593. https://doi.org/10.1016/j.pneurobio.2010.11.005

Hirsch, J. (2005). An index to quantify an individual's scientific research output. Proceedings of the National Academy of Sciences, 102(46), 16569-16572. https://dx.doi.org/10.1073/pnas.0507655102

Holstein, M., \& Minkler, M. (2003). Self, society, and the "new gerontology". The Gerontologist, 43, 787-796. https://doi.org/10.1093/ geront $/ 43.6 .787$

Huang, Y., Macera, C. A., Blair, S. N., Brill, P. A., Kohl, H. W., \& Kronenfeld, J. J. (1998). Physical fitness, physical activity, and functional limitation in adults aged 40 and older. Medicine \& Science in Sports \& Exercise, 30, 1430-1435. https://doi.org/10.1097/00005768199809000-00013

Inouye, S. K., Studenski, S., Tinetti, M. E., \& Kuchel, G. A. (2007). Geriatric syndromes: clinical, research, and policy implications of a core geriatric concept. Journal of the American Geriatrics Society, 55, 780-791. https://doi.org/10.1111/j.1532-5415.2007.01156.x 
Katz, S., \& Calasanti, T. (2015). Critical perspectives on successful aging: does it "appeal more than it illuminates"? The Gerontologist, 55, 26-33. https://doi.org/10.1093/geront/gnu027

Klibanski, A., Adams-Campbell, L., Bassford, T. L., Blair, S. N., Boden, S. D., Dickersin, K., ... Russell, W. E. (2001). Osteoporosis prevention, diagnosis, and therapy. Journal of the American Medical Association, 285, 785-795.

Kligman, A. M. (1979). Perspectives and problems in cutaneous gerontology. The Journal of Investigative Dermatology, 73, 39-46. https://doi.org/10.1111/1523-1747.ep12532758

Krause, K. H. (2007). Aging: a revisited theory based on free radicals generated by NOX family NADPH oxidases. Experimental Gerontology, 42, 256-262. https://doi.org/10.1016/j.exger.2006.10.011

Krekula, C. (2007). The intersection of age and gender - reworking gender theory and social gerontology. Current Sociology, 55, 155-171. https://doi.org/10.1177/0011392107073299

Kvelde, T., McVeigh, C., Toson, B., Greenaway, M., Lord, S. R., Delbaere, K., \& Close, J.C. T. (2013). Depressive symptomatology as a risk factor for falls in older people: systematic review and meta-analysis. Journal of the American Geriatrics Society, 61, 694-706. http://doi.org/10.1111/jgs.12209

Laforest, J. (1991). Introducción a la gerontología. Barcelona: Herder.

Luchetti, M., Terracciano, A., Stephan, Y., \& Sutin, A. R. (2016). Personality and cognitive decline in older adults: data from a longitudinal sample and meta-analysis. Journals of Gerontology - Series B Psychological Sciences and Social Sciences, 71, 591-601. http://doi.org/10.1093/geronb/gbu184.

Lui, C.W., Everingham, J.A., Warburton, J., Cuthill, M., \& Bartlett, H. (2009). What makes a community age-friendly: a review of international literature. Australasian Journal of Ageing, 28, 116-121. https://doi.org/10.1111/j.1741-6612.2009.00355.x

Martínez, M. A., Herrera, M., López-Gijón, J., \& Herrera-Viedma, E. (2014). H-Classics: Characterizing the concept of citation classics through H-index. Scientometrics, 98, 1971-1983. http://doi.org/10.1007/s11192-013-1155-9

Martinson, M., \& Berridge, C. (2014). Successful aging and its discontents: a systematic review of the social gerontology literature. The Gerontologist, 55,58-69. https://doi.org/10.1093/geront/gnu037

Masi, C. M., Chen, H., \& Hawkley, L. C., \& Cacioppo, J. T. (2011). A meta-analysis of interventions to reduce loneliness. Personality and Social Psychology Review, 15, 219-266. http://doi.org/ 10.1177/1088868310377394

Menec, V., Means, R., Keating, N., Parkhurst, G., \& Eales, J. (2011). Conceptualizing age-friendly communities. Canadian Journal on Aging / La Revue Canadienne Du Vieillissement, 30, 479-493. https://doi.org/10.1017/S0714980811000237

Morley, J.E., Vellas, B., van Kan, G. A., Anker, S. D., Bauer, J. M., Bernabei, R., ... \& Fried, L.P. (2013). Frailty consensus: a call to action. Journal of the American Medical Directors Association, 14, 392-397. https://doi.org/10.1016/j.jamda.2013.03.022

Navarro, M. (2019). Viejas en el género. En P. Danel, M. Navarro (Comp.), La gerontología será feminista (pp. 43-70). Buenos Aires: Editorial Fundación La Hendija.

Nelson, M. E., Rejeski, W. J., Blair, S. N., Duncan, P. W., Judge, J. O., King, A. C., ... \& Castaneda-Sceppa, C. (2007). Physical activity and public health in older adults: recommendation from the American College of Sports Medicine and the American Heart Association. Circulation, 116, 1094. https://doi.org/10.1161/CIRCULATIONAHA.107.185650

OEA. (2015). Convención interamericana sobre la protección de los derechos humanos de las personas mayores. New York: Organización de Estados Americanos. Recuperado desde http://www.oas.org/es/sla/ddi/docs/tratados_multilaterales_interamericanos _A-70_derechos_humanos_personas_mayores.pdf

Ong, A. D., Uchino, B. N., \& Wethington, E. (2016). Loneliness and health in older adults: a mini-review and synthesis. Gerontology, 62, 443-449. http://doi.org/10.1159/000441651

Orellana, V. (2013). Introducción a la gerontología social y conceptos básicos de geriatría. Santiago: Pontificia Universidad Católica de Chile.

Oswald, F., Wahl, H. W., Schilling, O., Nygren, C., Fänge, A., Sixsmith, A., ... \& Iwarsson, S. (2007). Relationships between housing and healthy aging in very old age. The Gerontologist, 47, 96-107. https://doi.org/10.1093/geront/47.1.96

Paola, J. (2019). Relaciones conceptuales y desenvolvimientos del Trabajo Social en el Campo Gerontológico. Revista Pensamiento y Acción Interdisciplinaria, 5, 29-45. https://doi.org/10.29035/pai.5.1.29

Paredes, M., Lladó, M., \& Pérez, R. (2017). La construcción de interdisciplina en el campo del envejecimiento en Uruguay. Revista Interdisciplina, 5, 135-160. http://dx.doi.org/10.22201/ceiich.24485705e.2017.13.62391

Phillipson, C. (2007). The 'elected' and the 'excluded': Sociological perspectives on the experience of place and community in old age. Ageing and Society, 27, 321-342. https://doi.org/10.1017/S0144686X06005629

Pinquart, M., \& Sörensen, S. (2003). Differences between caregivers and noncaregivers in psychological health and physical health: a meta-analysis. Psychology and Aging, 18, 250-267. http://doi.org/10.1037/0882-7974.18.2.250 
Pinquart, M., \& Sörensen, S. (2007). Correlates of physical health of informal caregivers: a meta-analysis. Journals of Gerontology Series B Psychological Sciences and Social Sciences, 62, 126-137. http://doi.org/10.1093/geronb/62.2.P126

Piña, M. (2015). La formación del espíritu científico en el trabajo social y la vigilancia epistemológica en el campo gerontológico. En J. Paola, M. Tordo, \& P. Danel (Comp.), Más mayores, más derechos. Diálogos interdisciplinarios sobre vejez (pp. 227-258). La Plata: Editorial de la Universidad Nacional de la Plata.

Piña-Morán, M., \& García, L. (2016). Socio-cultural connections and ruptures: social roles of older adults in Chile. Journal of Population Ageing, 9, 263-280. https://doi.org/10.1007/s12062-016-9142-6

Piña, M., \& Gómez, V. (2019). Envejecimiento y género: reconstruyendo los roles sociales de las personas mayores en los cuidados. Revista Rupturas, 9, 23-38. https://doi.org/10.22458/rr.v9i2.2521

Piña-Morán, M., Olivo-Viana, M.G., Martínez-Maldonado, M.L., \& Mendoza-Núñez, V.M. (2018). Intervención situacional gerontológica: estrategias para potenciar los roles sociales de los mayores. Revista Médica del Instituto Mexicano del Seguro Social, 56, 102-109.

Polanco-Carrasco, R. (2016). La publicación científica como placebo. Cuadernos de Neuropsicología - Panamerican Journal of Neuropsychology, 10, 8-12. https://doi.org/10.7714/cnps/10.2.101

Polanco-Carrasco, R. (2017). ¿Quién es el responsable del éxito o fracaso de una publicación? Cuadernos de Neuropsicología - Panamerican Journal of Neuropsychology, 11, 11-12. https://oi.org/10.7714/CNPS/11.3.101

Rolland, Y., Dupuy, C., van Kan, G. A., Gillette, S., \& Vellas, B. (2011). Treatment strategies for sarcopenia and frailty. Medical Clinics, 95, 427-438. https://doi.org/10.1016/j.mcna.2011.02.008

Rolland, Y., van Kan, G. A., Gillette-Guyonnet, S., \& Vellas, B. (2011). Cachexia versus sarcopenia. Current Opinion in Clinical Nutrition \& Metabolic Care, 14, 15-21. https://doi.org/10.1097/MCO.0b013e328340c2c2

Roqué, M. R., Salvà, A., Bolíbar, I., \& Rivero, T. (2012). Tendencias en salud percibida y dependencia de la población mayor española: evolución entre los años 1993 y 2006. Medicina Clínica, 139, 284-289. https://doi.org/10.1016/j.medcli.2011.06.021

Salvà, A. (2012). El mini nutritional assessment. Veinte años de desarrollo ayudando a la valoración nutricional. Revista Española de Geriatría y Gerontología, 47, 245-246. https://doi.org/10.1016/j.regg.2012.10.001

Schulz, R., \& Heckhausen, J. (1996). A life span model of successful aging. American Psychologist, 51, 702. https://psycnet.apa.org/ doi/10.1037/0003-066X.51.7.702

Seeman, T. E. (2000). Health promoting effects of friends and family on health outcomes in older adults. American Journal of Health Promotion, 14, 362-370. https://doi.org/10.4278/0890-1171-14.6.362

Shen, C., Nguyen, D. T., \& Hsu, P. (2019). Bibliometric networks and analytics on gerontology research. Library Hi Tech, 37, 88-100. http://doi.org/10.1108/LHT-11-2017-0247

Sui, X., LaMonte, M. J., Laditka, J. N., Hardin, J. W., Chase, N., Hooker, S. P., \& Blair, S. N. (2007). Cardiorespiratory fitness and adiposity as mortality predictors in older adults. JAMA, 298, 2507-2516. https://doi.org/10.1001/jama.298.21.2507

Tolson, D., Rolland, Y., Andrieu, S., Aquino, J.P., Beard, J., Benetos, A., ... \& The International Association of Gerontology and Geriatrics/World Health Organization/Society Française de Gérontologie et de Gériatrie Task Force. (2011). International Association of Gerontology and Geriatrics: A Global Agenda for Clinical Research and Quality of Care in Nursing Homes. Journal of the American Medical Directors Association, 12, 184-189. https://doi.org/10.1016/j.jamda.2010.12.013

Twigg, J. (2007). Clothing, age and the body: a critical review. Ageing \& Society, 27, 285-305. https://doi.org/10.1017/S0144686X06005794

United Nations. (1983). Viena international plan of action on ageing. New York: UN. Recuperado desde https://www.un.org/en/events/ elderabuse/pdf/vipaa.pdf

United Nations. (2002). Political declaration and Madrid international plan of action on ageing. New York: UN. Recuperado desde https://www.un.org/en/events/pastevents/pdfs/Madrid_plan.pdf

Van Leeuwen, T. N. (2004). Second generation bibliometric indicators: the improvement of existing and development of new bibliometric indicators for research and journal performance assessment procedures. Leiden: Universiteit Leiden.

Vanegas, A., Messa, J., Cardoza, D., Tovar, J., \& Ocampo, A. (2018). Ambientes enriquecidos, sujetos geriátricos y procesos de memoria; un acercamiento a los procesos cognitivos en la edad adulta. Cuadernos de Neuropsicología - Panamerican Journal of Neuropsychology, 12(2), 1-37. https://doi.org/10.7714/cnps/12.2.201

Vellas, B., Coley, N., Ousset, P. J., Berrut, G., Dartigues, J. F., Dubois, B., ... \& Touchon, J. (2012). Long-term use of standardised Ginkgo Biloba extract for the prevention of Alzheimer's disease (GuidAge): a randomised placebo-controlled trial. The Lancet Neurology, 11, 851-859. https://doi.org/10.1016/S1474-4422(12)70206-5

Vergara, J. (2014). Epistemología. En R. Salas (Coord.), Pensamiento crítico latinoamericano: conceptos fundamentales (pp. 267-291). Santiago de Chile: Ediciones UCSH.

Wahl, H., \& Weisman, G., (2003). Environmental gerontology at the beginning of the new millennium: reflections on its historical, empirical, and theoretical development. The Gerontologist, 43, 616-627. https://doi.org/10.1093/geront/43.5.616 
Weindruch, R., Kayo, T., Lee, C. K., \& Prolla, T. A. (2001). Microarray profiling of gene expression in aging and its alteration by caloric restriction in mice. The Journal of Nutrition, 131, 918S-923S. https://doi.org/10.1093/jn/131.3.918S

Wiles, J. L., Allen, R. E., Palmer, A. J., Hayman, K. J., Keeling, S., \& Kerse, N. (2009). Older people and their social spaces: a study of well-being and attachment to place in Aotearoa New Zealand. Social Science \& Medicine, 68, 664-671. https://doi.org/10.1016/j. socscimed.2008.11.030.

Wiles, J., Leibing, A., Guberman, N., Reeve, J., Allen, R., (2012). The meaning of "aging in place" to older people. The Gerontologist, 52, 357-366. https://doi.org/10.1093/geront/gnr098.

Woo, J., Leung, J., \& Morley, J. E. (2012). Comparison of frailty indicators based on clinical phenotype and the multiple deficit approach in predicting mortality and physical limitation. Journal of the American Geriatrics Society, 60, 1478-1486. https://doi. org/10.1111/j.1532-5415.2012.04074.x

Yuni, J. (2019). Prólogo. En P. Danel, \& M. Navarro (Comp.), La gerontología será feminista (pp. 15-22). Buenos Aires: Editorial Fundación La Hendija.

Yuni, J., \& Urbano, C. (2008). Envejecimiento y género: perspectivas teóricas y aproximaciones al envejecimiento femenino. Revista Argentina de Sociología, 6, 151-169. 